BEATA SPIERALSKA-KASPRZYK

CARDINAL STEFAN WYSZYNSKI UNIVERSITY

IN WARSAW

\title{
LES DÉRIVÉS DÉNOMINAUX LATINS EN -MONIUM
}

Les dérivés en -monia et -monium ne sont pas très nombreux. F.T. Cooper ${ }^{1}$, citant les travaux de K. Paucker qui en a répertoriés $31^{2}$ (sauf caerimonia) indique que seulement 11 noms de ce groupe sont employés par Cicéron, et que parmi les autres il y en a 4 terminés en -monia contre 16 terminés en -monium. Un lexème manque sur la liste donnée par Cooper, à savoir flamonium. Par ailleurs ce groupe des noms est aussi diversifié selon d'autres critères. On peut, par exemple, diviser tous ces substantifs en trois types selon la base dérivationnelle : il y a des dérivés à base verbale, nominale et adjectivale.

En ce qui concerne leur signification, les déverbatifs et les dérivés déadjectivaux se laissent assez aisément analyser : il s'agit des noms d'action, comme querimonia « l'action de se plaindre » ou des noms des traits, comme sanctimonia « sainteté ».

Quant aux dénominatifs, ils présentent des difficultés. En latin classique, attestés chez Cicéron, on trouve quatre noms de ce type : matrimonium, patrimonium, testimonium et vadimonium, tous les quatre appartenant clairement à la langue juridique. À ce nombre ajoutons encore mercimonium, mendicimonium, moechimonium, regimonium (quoique celui-ci puisse aussi bien être un déverbatif ${ }^{3}$ ), fratrimonium, pactimonium, flamonium, litimonium, et, en latin médieval, abmatrimonium et forimatrimonium. Tous ces noms se distinguent aussi des dérivés

\footnotetext{
1 Cooper 1895: 36-37.

2 Paucker 1884: 4, n. 3.

3 Le dictionnaire de Forcellini, par exemple, définit ce lexème comme le nomen verbale. Cf. Forcellini, Furlanetto, De Vit 1871: s.u.
} 
déverbaux et déadjectivaux par le fait qu'ils n'ont pas de doublets en -monia, tandis que querimonium et sanctimonium, par exemple, sont concurrencés par les doublets féminins querimonia et sanctimonia. À ce répertoire ajoutons encore un nom bien distinct, à savoir caerimonium, dont le sens est clair, mais l'étymologie inconnue. Peut-être le fait qu'il existe un doublet caerimonia suggère qu'il faudrait considérer ce dérivé comme construit à partir d'un adjectif ou d'un verbe.

Quelle que soit leur base dérivationnelle, on considère en général que tous ces noms sont formés à l'aide des suffixes combinés mōn-ioet $m \bar{o} n-i a^{4}$. F. Bopp appelle ces substantifs des Doppelsuffix Abstracta ${ }^{5}$.

Mis à part quatre termes juridiques attestés chez Cicéron, quatre autres noms, parmi ceux que j'ai énumérés, sont attestés chez des auteurs de l'antiquité : flamonium, mercimonium, mendicimonium et moechimonium. Six autres (regimonium, fratrimonium, pactimonium, litimonium, abmatrimonium et forimatrimonium) sont tardifs. Les deux noms, mendicimonium et moechimonium sont des créations lexicales humoristiques :

Quod Laberius uerba pleraque licentius petulantiusque finxit; et quod multis item uerbis utitur, de quibus, an sint Latina, quaeri solet. Laberius in mimis, quos scriptitauit, oppido quam uerba finxit praelicenter. Nam et 'mendicimonium' dicit et 'moechimonium' et 'adulterionem' 'adulteritatem 'que pro 'adulterio' et 'depudicauit' pro 'stuprauit' et 'abluuium' pro 'dilunio'

Que Laberius a inventé plusieurs mots, de manière libre et impudente ; et qu'il emploi beaucoup de mots dont on se demande, s'ils sont latins. Laberius, dans ses mimes, qu'il avait habitude d'écrire, a inventé des mots de manière extrêmement licencieuse. Il dit en fait " mendicimonium » et " moechimonium » et " adulterionem » ou " adulteritatem 》 au lieu de " adulterium ", et " depudicauit 》 au lieu de "stupranit » et " ablunium » au lieu de « dilunio ». (Noctes Atticae 16, 7, 2).

Cela nous laisse, pour l'époque classique, en présence de six dénominatifs ayant une signification bien précise. Mais avant de nous

4 Cette observation est d'ailleurs ancienne. On la trouve entre autre dans: Donaldson 1860: 480.

$5 \quad$ Cf. Bopp 1842: 1111. 
occuper de cette signification, regardons de plus près deux de ces six noms.

Il faut d'abord remarquer que cinq substantifs sont indubitablement formés sur la base qui est un nom de personne : flamen $>$ flamonium, mater $>$ matrimonium, pater $>$ patrimonium, testis $>$ testimonium, et uas $>$ uadimonium.

Le premier d'entre eux pose cependant quelques difficultés. Tout d'abord, il faut restituer une forme non attestée flaminimonium, et ensuite c'est le seul parmi les lexèmes cités qui soit apparenté à un adjectif flamonius, - $a$, -um. L'existence de ce dernier permet même de contester l'étymologie avec le suffixe -monium. Il est en effet possible que le nom flamonium soit un adjectif substantivé. On mettrait alors flamonium sur le même plan que cauponium (synonyme de caupona) apparenté à l'adjectif cauponius,-a,-um (attesté déjà chez Plaute) ou les adjectifs tels que lenonius (de même attesté chez Plaute) et fullonius (attesté chez Caton). La difficulté à laquelle on se heurte en acceptant cette hypothèse c'est la voyelle -o- qui appartient au nom de base dans cauponius, lenonius et fulonius, mais qui est absente dans le nom de flamen. Il faut cependant souligner que l'adjectif flaminius (avec le vocalisme attendu) est bien attesté. Il est, bien sûr, le synonyme de flamonius. Le dictionnaire de P. Glare cite également le substantif flaminium, avec cette restriction cependant qu'il s'agit d'une variante douteuse de flamonium, présente dans quelques manuscrits. Toutes ces donnés appuient, à mon avis, des doutes concernant l'étymologie de flamonium. Au lieu d'y voir un dérivé au suffixe -monium on peut partir d'un adjectif, substantivé ensuite, formé à l'aide du suffixe -ius : flaminius. Son doublet avec la voyelle -o- serait analogique, d'un côté aux adjectifs tels que cauponius, lenonius ou fullonius, de l'autre à tous les noms en -monium (qu'ils soient les dérivés dénominaux, déverbaux ou déadjectivaux).

Ayant donc mis à part le nom flamonium, on reste avec cinq lexèmes, dont quatre sont formés sur les noms de personne : matrimonium, patrimonium, testimonium, uadimonium, tandis que le cinquième semble formé sur le nom de chose : mercimonium, venant, comme l'indique Glare, de merx auquel s'est ajouté le suffixe -monium. 
Mais ce terme présente encore une autre difficulté, d'ordre sémantique cette fois-ci. C'est qu'il est, apparemment, le synonyme exact de merx, les deux signifiant « la marchandise ». Le procédé de former un nom sur un autre nom sans qu'aucune variation sémantique n'y intervienne (ne serait-ce que la connotation hypocoristique) est assez répandu en latin tardif, quand on employait ce stratagème pour transférer des lexèmes des paradigmes moins réguliers (et plus difficiles) vers ceux qui était plus régulier et, par là, plus productifs. C'est ainsi qu'on a créé litimonium, décliné selon la deuxième déclinaison, pour remplacer lis de la troisième déclinaison. Mais ce même procédé est surprenant en latin archaïque (mercimonium étant attesté déjà chez Plaute). Je crois qu'il est plus logique d'expliquer ce nom comme formé sur la base verbale. Tout comme alimonium et alimonia venant de alo, gaudimonium venant de gaudeo, parcimonia venant de parco, querimonium et querimonia venant de queror, ou tardif plangimonium venant de plango. Mercimonium viendrait donc de mercor ce qui expliquerait la synonymie avec merx. On aurait en fait deux noms : un nom racine et un dérivé déverbal (la situation serait donc analogique à la coexistence de donum et du déverbatif donatio, ayant une signification très proche). La sémantique aussi serait alors claire : mercimonium signifierait ce qui est l'objet de mercor, « la marchandise », analogiquement à alimonium qui désigne 1'objet de alo, « la nourriture ». En admettant cette hypothèse, on classifie donc le nom mercimonium parmi les déverbatif et on ne garde que quatre dénominatifs classiques : matrimonium, patrimonium, testimonium et uadimonium.

Quant au sens de ces quatre noms, la plus grande difficulté vient du fait que la signification du suffixe -monium semble moins cohérente que ne le veulent certains chercheurs. Je crois que Donaldson a bien raison, en disant que la signification véhiculée par les noms en -monium est loin d'être consistante: «while patrimonium means 'a paternal estate', matrimonium signifies 'lawful wedlock', and while testimonium and uadimonium denote that which is given by a testis or uas, mercimonium is not distinguishable in meaning from the simple word merx $»^{6}$. Les deux significations sont expliquées ainsi par M. Fruyt : « The Latin lexicon also contains (...) fundamental lexemes built with suffixes. One

6 Donaldson 1860: 480-481. 
of the most important of these is -(i)monium neut. (joining -mōn- and -ium from *-yo-neut.), documented within the social and legal vocabulary in patr-imonium 'property of a pater familias' (...), matr-imonium 'state of being married' for a woman. $»^{7}$

J.T. White par contre n'éprouve pas cette difficulté. Selon lui, les dérivés formés sur les substantifs signifient originellement l'appartenance ${ }^{8}$. À le croire, patrimonium signifierait « ce qui appartient au père », matrimonium - « ce qui appartient à la mère », et testimonium - « ce qui appartient au témoin ». Cette explication n'est pas acceptable à mon avis.

E. Benveniste, quant à lui, offre l'explication suivante : "Pris littéralement matrimonium signifie 'condition légale de mater', conformément à la valeur des dérivés en -monium, qui sont tous des termes juridiques (testimonium, uadimonium, mercimonium, et naturellement patrimonium) ». Un peu plus loin Benveniste remarque que le terme matrimonium n'est pas analogique au patrimonium et explique que « matrimonium définit la condition à laquelle accède la jeune fille : celle de mater (familias) $»$. Certaines de ces observations sont quand même discutables. Le terme mercimonium n'est pas plus juridique que merx, et, bien que l'interprétation de matrimonium semble tout à fait juste, cela n'explique pas, pourquoi le patrimonium ne signifie pas la 'condition à laquelle accède le jeune homme'.

J. Daude étudie les noms en -monium parmi d'autres substantifs abstraits de qualité, et il précise que ces lexèmes désignent les « conditions sociales : flamonium 'diginité de flamine', matrimonium et patrimonium 'statut de mère, statut de père' (initialement), testimonium et uadimonium 'qualité de témoin, de caution' (...) alimonium, mercimonium 'valeur de nourriture, de marchandise' $\gg{ }^{10}$. Dans ce répertoire il met cependant sur le même plan des dérivés dénominaux et déverbaux. Plus loin il ajoute cette observation sur le suffixe lui-même :

«L'élément -mon- rappelle le suffixe IE de nom d'agent *-me/ on- et on a supposé gratuitement que des formes comme alimonia,

Fruyt 2001: 163.

Cf. White 1858: 100.

Benveniste 1969: 243.

10 Daude 2002: 233. 
caerimonia (...) impliquaient des bases $\operatorname{alimo}(n),{ }^{*}$ caerimo(n) (...) de noms d'agent. (...) On se bornera à constater qu'en latin, le suffixe est insécable $\gg{ }^{11}$.

Le manque de symétrie entre matrimonium et patrimonium était si frappant qu'il a engendré parfois des curieuses spéculations. R. Metzner, par exemple, dans un livre consacré à l'étude des mythes, présente des hypothèses qui, de point de vue linguistique, sont tout simplement fausses : "With the centuries-long transition to patriarchy and male dominance came the emphasis on the patrilineal inheritance of property (...). The substitution of patrilineal for matrilineal inheritance is reflected in the asymmmetrical meanings of the words patrimony and matrimony. The Latin suffix -monium, possibly related to moneta 'money', refers to property, and patrimony now refers to property or legacy inherited from the father. However, matrimony, literally 'maternal property', now refers only to the marriage alliance itself, meaning something like 'legal maternity' ${ }^{12}$. Le suffixe -monium, bien sûr, n'a rien à voir avec le nom moneta, qui d'ailleurs ne signifie 'l'argent, la monnaie' que par une évolution sémantique bien particulière : l'institution romaine fabriquant les pièces de monnaie a pris son nom du temple de Iuno Moneta se trouvant dans son voisinage.

$\mathrm{Ce}$ qu'écrit à propos de ces termes $\mathrm{C}$. Francese est plus juste : « $\mathrm{Pa}$ trimonium fuses the root for 'father', patr-, with the fairly common abstract noun suffix -monium (...). The obvious counterpart of patrimony would be matrimony, combining the same suffix with the root for 'mother'. But in Latin, as in English, there is a striking assymetry between the two. Matrimonium means marriage, but only for a woman. (...) Patrimonium means not marriage or fatherhood but inherited property and fortune (...). Patrimonium and matrimonium reflect the assymetrical roles and duties of father and mother in the Roman patriarchal family $»^{13}$.

Ces observations sont tout à fait pértinentes, mais insuffisantes pour découvrir le sens exact du suffixe -monium. Si, en fait, matrimonium signifie 'la condition légale de mère', quelque chose comme la

\footnotetext{
11 Daude 2002: 233.

12 Metzner 1994.

13 Francese 2007: 89.
} 
'maternité légale', patrimonium devrait signifier 'la condition légale de père', la 'paternité légale'. Mais il n'en est rien, puisque le patrimonium désigne une chose bien concrète, l'héritage, des biens matériels que le père laisse aux enfants. Et testimonium n'est certainement pas une 'condition de témoin', ni uadimonium 'condition d'un garant'. Ces deux derniers termes signifient bien : 'ce que le témoin donne' et 'ce que le garant donne'.

On peut donc relever une certaine symétrie dans la signification de ces derniers trois lexèmes ce qui suggère que le sens du suffixe -monium était 'la chose donnée par X' ou 'la chose que l'on reçoit de la part de X'. Il n'y a que matrimonium qui semble incompatible avec ce sens. Mais peut-être l'analyse juridique de cette institution peut apporter une solution à cette difficulté.

«Investigation of the occurrence of the word matrimonium in classical Roman law reveals its use in four categories: first, the status of liberty for children; secondly, the restitution of dowry; thirdly, the restitution of gifts and donations at the end of a relationship; and fourthly, the decreeing of divorce. (...) The first category of matrimonial cases concerned liberty. Gaius linked the status of liberty to matrimonium. Children born and educated in a high-quality relationship received liberty at birth. Further, the children of a slave living in high-quality matrimonial relationship with her dominus also received the status of liberty. The three other categories all required the judge to deal with the end of matrimonium ${ }^{14}$.

Il y a donc quatre traits caractéristiques du matrimonium, dont trois concernent la rupture de cet état légal. Un seul trait qui est lié à son existence a de l'importance du point de vue des enfants. Il faut ici souligner que patrimonium aussi est bien une institution cruciale du point de vue des enfants, de même que testimonium l'est du point de vue d'une des parties concernées par le procès verbal, et de même que uadimonium l'est du point de vue de la personne ayant besoin d'une garantie. Toutes ces personnes (enfants, des parties du procès verbal et ceux qui ont besoin d'une garantie légale) sont donc des bénéficiaires, des récipients. Dans le cas de patrimonium le récipient est la même personne du point de vue de laquelle le pater est justement pater. Analogiquement,

14 Waelkens 2010. 
à croire Waelkens, le récipient du matrimonium est la même personne du point de vue de laquelle la mater est justement mater, c'est à dire l'enfant.

Ces données nous permettent donc de proposer une nouvelle interprétation prétation du mot matrimonium. Il s'agirait, analogiquement au patrimonium de 'ce que la mère donne'. En recevant le patrimonium l'enfant reçoit de la part de son père les biens matériaux; en recevant le matrimonium l'enfant reçoit de la part de sa mère, si elle est légalement mariée, le statut d'homme libre. Le sens que matrimonium reçoit au Moyen Âge, à savoir celui de 'l'héritage de la part de la mère'15, ne serait donc pas uniquement l'effet d'une évolution établissant la symétrie là, où elle manquait, mais aussi le développement du sème déjà présent.

Contre cet hypothèse on pourrait argumenter que l'emploi du lexème en question dans les expressions du type in matrimonium ducere indiquent qu'il est centré principalement autour de la personne d'une femme qu'on épouse, non pas autour de ses futurs enfants. Mais cet argument peut facilement être contesté : le lexème mater est incontestablement présent dans matrimonium, les futurs enfants sont donc explicitement présents dans ce substantif.

Si les quatre dérivés dénominatifs en -monium, appartenant au lexique judiciaire, partagent tous le sens de 'la chose donnée par', on pourrait se demander si l'élément -monium ne peut recevoir une nouvelle interprétation. Ce sens en effet rapproche -monium du nom munus. Dans le Dictionnaire Étymologique de la Langue Latine on trouve en effet l'explication suivante du substantif munia: " mūnia, -ium (arch. moenia) pl. n.: même sens que münera 'fonctions officielles, devoirs, charges d'un magistrat'. (...) Sur mūnia a été bâti un nominatif sg. münium qu'on trouve dans les gloses, traduit par $\lambda \varepsilon \imath \tau$ ovprí $\alpha$ CGL II 504, 37; 361, 40. (...) Münia est un archaïsme de la langue officielle $»^{16}$.

Je propose donc de considérer quatre noms: patrimonium, matrimonium, testimonium et uadimonium (qui appartiennent tous à la langue officielle) comme des noms composés, avec - mūnium comme deuxième élément. Le vocalisme - $o$ - serait analogique aux noms déverbaux

15 On trouve aussi, en latin médiéval, le substantif fratrimonium signifiant 'l'héritage de la part du frère'.

16 Érnout, Meillet 1951: s.u. 
et déadjectivaux avec le suffixe -monium, ces derniers n'appartenant aucunement à la langue officielle.

\section{BIBLIOGRAPHIE}

Benveniste E., 1969, Le vocabulaire des institutions indo-européennes, Paris. Bopp F., 1842, Vergleichende Grammatik des Sanskrit, Zend, Griechischen, Lateinischen, Litthauischen, Altslawischen, Gothischen und Deutschen, Berlin.

Cooper F. T., 1895, Word formation in the Roman sermo plebeius, New York.

Daude J., 2002, 'Les substantifs abstraits de qualité', [dans:] C. Kircher-Durand (éd.), Grammaire fondamentale du latin, tome IX. Création lexicale: la formation des noms par dérivation suffixale, Louvain, pp. 225-305.

Donaldson J. W., 1860, Varronianus. A Critical and Historical Introduction to the Ethnography of Ancient Italy and to the Philological Study of the Latin Language, London.

Érnout A., Meillet A., 1951, Dictionnaire Étymologique de la langue latine, Paris.

Forcellini E., Furlanetto G., De Vit V., 1871, Totius Latinitatis lexicon. Prati.

Francese C., 2007, Ancient Rome in so many words, New York.

M. Fruyt, 2001, 'Word-Formation in Classical Latin', [dans:] J. Clackson (éd.), A Companion to the Latin Language, Oxford, pp. 157-175.

Metzner R., 1994, The Well of Remembrance. Rediscovering the Earth Wisdom Myths of Northern Europe, Boston.

Paucker K., 1884, Materialien Zur Lateinischen Worterbildungsgeschichte, Bd. 2, Berlin.

Waelkens L.L.J.M., 2010, 'Medieval Family and Marriage Law: From Actions of Status to Legal Doctrine', [dans:] J. W. Cairns, P. J. du Plessis (éds.), The Creation of the Ius Commune: From Casus to Regula, Edinburgh, pp. 103-126.

White J.T., 1858, Latin suffixes, London. 